\title{
LOCAL QUOTIENT RINGS ${ }^{1}$
}

\author{
M. SATYANARAYANA
}

1. Introduction. In the works of Goldie [6] and [7], Small [9] and Talintyre [11] and [12] one finds the conditions for a ring to have a semisimple, simple or right Artinian right quotient rings. There also exist rings with right quotient ring as a local ring. The main aim of this paper is to investigate the conditions for a ring to have a local quotient ring and thus provide methods for the construction of noncommutative local rings.

Definitions. A ring $R$ with identity is called a LOCAL RING if $R$ has a unique maximal right ideal $M$ or equivalently, sum of any two nonunits is a nonunit. We denote the local ring by $(R, M)$. Trivially division rings are local rings.

A ring $R$ with identity is said to have a RIGHT QUOTIENT RING $Q$ if (i) $R \subseteq Q$, (ii) every nonzero divisor (regular element) in $R$ has a two-sided inverse in $Q$ and (iii) every $q \in Q$ can be written in the form $a b{ }^{-1}, a, b \in R, b$ is a nonzero divisor in $R$. In the commutative case this quotient ring is called the total quotient ring. It is well known that a ring $R$ has a quotient ring if it has a right multiple property, namely, for ' $a$ ' regular, $b \in R$, there exist $c, d$ in $R$ such that $a c=b d, d$ being regular.

Throughout this paper every ring is assumed to have a two-sided identity and unless otherwise specified quotient ring means a right quotient ring. We denote by $Z(R)$ or $Z$ and $J(R)$ or $J$ to represent the set of all zero-divisors and Jacobson radical in a ring $R$ respectively. It is shown in 2.4, that if a ring $R$ has a quotient ring $Q$, then $Q$ is a local ring iff $Z(R)$ is an ideal and thus the problem is reduced to proving the existence of quotient rings of rings with $Z$ as an ideal. We next consider the existence of quotient rings of the rings of the above type and we prove in 2.6 that rings with a weaker right multiple property and in 2.7 rings with $Z \subseteq J$ and with some additional hypothesis have quotient rings. In $\$ 3$ following the commutative case that any ring can be imbeddable in a local ring, we investigate the conditions for a ring imbedded in a local ring, to have a local quotient ring. In $\$ 4$ we discuss about the necessity of the conditions used in Theorem 2.7.

Received by the editors July 20, 1967.

1 This paper is a portion of the author's doctoral dissertation written at the University of Wisconsin under the direction of Professor Edmund $\mathrm{H}$. Feller. 


\section{Internal characterization.}

2.1 Proposition. If a ring $R$ has a local quotient ring, then $Z(R)$ is an ideal.

Proof. If $(Q, M)$ is a local quotient ring of $R$, then every zerodivisor of $R$ is in $M$. Hence $Z(R)=M \cap R$, is an ideal.

It can be seen in 2.2 and 2.3 that the condition that $Z$ is an ideal is sufficient in the commutative case but not in the noncommutative case.

2.2 Proposition. If $R$ is a commutative ring, then $R$ has a local (total) quotient ring iff $Z$ is an ideal.

Proof. By virtue of 2.1 , it suffices to show that if $Z$ is an ideal, then $R$ has a local quotient ring. Clearly $Z$ is a prime ideal and hence $S=\{a / b: a \in R, b \notin Z\}$ is a local ring. Since $R$ can be identified with the set of the elements of the form $a / 1, a \in R, R \subseteq S$. Evidently $S$ is the quotient ring of $R$.

2.3 Examples. Malcev's example of noncommutative integral domain not imbeddable in a division ring proves that 2.2 is not true in the noncommutative case. From this we can also construct an example for the case of nonintegral domains. Consider $S=\{a+b x$ : $a, b \in R$, where $R$ is Malcev's integral domain not imbeddable in a division ring $\}$, subject to the conditions $x^{2}=0, x r=r x$ for every $r \in R$. $S$ is a ring under the usual rules of addition, multiplication and equality defined for polynomials. Evidently $Z(S)=x R$ is an ideal. If $S$ has a quotient ring $Q$, then every nonzero element of $R$ is invertible in $Q$ and $D=\left\{a b^{-1}: a, b \in R, b \neq 0\right\}$ is a division ring. Because of the ring isomorphism $r \rightarrow r / 1, r \in R, R$ is imbedded in a division ring $D$, a contradiction.

However, if we assume that the ring has a quotient ring, then the condition that $Z$ is an ideal is necessary and sufficient for the quotient ring to be a local ring.

2.4 Proposition. Let $R$ be a ring with a quotient ring $Q$. Then $Q$ is a local ring iff $Z(R)$ is an ideal. Also $Z Q$ is the unique maximal right ideal of $Q$.

Proof. In view of 2.1 , it is sufficient to prove that $Q$ is a local ring if $Z(R)$ is an ideal. Let $M$ be a maximal right ideal of $Q$. Since $Q$ is a quotient ring, every element of $M$ is a zero-divisor. So $M \cap R$ $\subseteq Z(R)$. But $M=(M \cap R) Q$ [5, Lemma 1.1]. Thus $M \subseteq Z Q$ [write $Z$ for $Z(R)]$. Then $Z Q=M$ or $Z Q=Q$. If $Z Q=Q$, then for a unit $q$ of $Q$, $q=j c^{-1}, j \in Z$ [5, Corollary 5.2]. Since $q$ is a unit, $j$ should be regular, 
a contradiction. Thus $Q$ is a local ring with $Z Q$ as the unique maximal right ideal.

By virtue of the Example 2.3, we must require some more conditions in addition to the condition that $Z$ is an ideal in order to prove the existence of local quotient rings. So according to 2.4 , this problem is reduced to finding the conditions if $Z$ is an ideal. In this case we can show that the right multiple property (which is the necessary and sufficient condition for the existence of a quotient ring) can be weakened.

2.5 Definition. A set $S$ in a ring $R$ is said to have right multiple property if $a, b \in S$ and $a$ is regular then there exist $r$ and $s, s$ regular, such that $a r=b s$.

2.6 Proposition. Let $R$ be a ring in which the set of all regular elements has right multiple property. Then $R$ has a local quotient ring if $Z$ is an ideal.

Proof. Let $a$ be regular and $b \in Z$. Since $Z$ is an ideal, $a+b$ is regular. Now, by right multiple property of regular elements, we have $a x=(a+b) y$ where $y$ is regular i.e., $a(x-y)=b y$. Hence $R$ has a quotient ring $Q$. This implies that $Q$ is a local ring by virtue of 2.4.

Now we seek conditions on when the set of regular elements can have right multiple property. In order to obtain this property, we assume the condition that $Z \subseteq J$. This happens to be an important property enjoyed by many rings with local quotient rings.

2.7 THEOREM. Let $R$ be a ring satisfying the following conditions:

(i) $Z$ is an ideal.

(ii) $Z \subseteq J$.

(iii) Finitely generated right ideals containing regular elements are principal.

Then $R$ has a local quotient ring.

Proof. Let $a$ and $b$ be regular in $R$. By the condition (iii), $a R+b R$ $=d R$. Here $d$ is regular since otherwise if $d \in Z, d R \subseteq Z$ and hence $a \in Z$, a contradiction. Now we have $a p+b q=d ; a=d a_{1}$ and $b=d b_{1}$. This implies $a_{1}$ and $b_{1}$ are regular since $Z$ is an ideal. Now $a p+b q$ $=d \Rightarrow a p a_{1}+b q a_{1}=d a_{1}=a \Rightarrow a\left(1-p a_{1}\right)=b q a_{1}$. If $q$ is regular, then $q a_{1}$ is regular. Hence $a$ and $b$ have right multiple property. Suppose $q \in Z$. Then $b q a_{1} \in Z$. Thus $a\left(1-p a_{1}\right) \in Z$. This implies $1-p a_{1} \in Z$ since $a$ is regular. Therefore $1-p a_{1} \in J$, since $Z \subset J$. Thus $p a_{1}$ is a unit i.e., $x a_{1}=1$. If $a_{1} x \neq 1$, then $x\left(a_{1} x-1\right)=0$. This implies $x \in Z$ i.e., $1 \in Z$, a contradiction. Thus $a_{1}$ has a two-sided inverse. Since $a=d a_{1}, d=a a_{1}^{-1}$ and so $d R \subset a R$. Therefore $b R=a R$ and $b(1)=a x$, where $x$ is regular. 
Thus the right multiple property is satisfied. Then it follows from 2.6 that $R$ has a local quotient ring.

The assumption of $Z \subseteq J$ in the hypothesis of 2.7 appears to be reasonable in view of von Neumann regular ring, which satisfies (iii) but not (i) and (ii) of the hypothesis of 2.7. But the ring of entire functions over the field of complex numbers satisfies the hypothesis of 2.7. This is a non-Noetherian ring. By passage to a residue class ring modulo a primary ideal, we have an example for which $Z \neq 0,[10]$.

An immediate application of 2.7 gives the following corollaries.

2.8 Corollary. Let $R$ be an integral domain in which finitely generated right ideals are principal. Then $R$ has a quotient skewfield.

2.9 Corollary [Cohn]. Any right Bezout ring [2, Theorem 5.2] has a quotient skewfield.

2.10 THEOREM. Let $R$ be a ring satisfying the following conditions:

(i) $Z$ is an ideal.

(ii) $Z \subseteq J$.

(iii) Right and left ideals containing regular elements are principal. Then $R$ has a right and left quotient ring $Q$ and $Q_{n}$ (the ring of $n \times n$ matrices over $Q$ ) is a right and left quotient ring of $R_{n}$.

Proof. By 2.7, $R$ has both right and left quotient rings. This implies that $R$ has a two-sided quotient ring $Q$.

Since $Z$ is an ideal, $a$ is regular iff $a+Z$ is regular in $R / Z$. By the conditions (i) and (iii), $R / Z$ is a two-sided principal ideal domain. Hence by 1.2 and 2.3 of [3], $Q_{n}$ is a two-sided quotient ring of $R_{n}$.

3. External characterization. In commutative ring theory, there is a natural construction of over rings as local rings. If $R$ is a commutative ring with identity and if $P$ is a prime ideal, then the fractions $a / b, a \in R, b \notin P$ form a local ring, which contains a copy of $R$. This type of construction of over rings as local rings appears to be not feasible for all noncommutative rings. But a start has been made in the work of Amitsur [1]. However, in the noncommutative case, we can construct local over rings of local rings. If $(R, M)$ is a local ring, consider $R[x]$ with the similar construction as in 2.3. Then $R[x]$ is a local ring with the unique maximal right ideal generated by $M$ and $X$. Now in this section we discuss when can a ring imbedded in a local ring, have a proper local quotient ring, as this is of frequent occurrence in the noncommutative case also.

3.1 Proposition. Let $R$ be a ring with a local quotient ring $(Q, M)$. Then (i) $Z(R)=M \cap R$ and (ii) $\bar{R}=R / Z(R)$ is a right Ore domain. 
Proof. Evidently $M$ contains all nonunits in $Q$. Hence $Z(R)$ $=M \cap R$ is an ideal in $R$. Moreover it can be seen readily that $\bar{R}$ is imbedded in $Q / M$ and $Q / M$ is the quotient field of $\bar{R}$.

3.2 Theorem. Let $R$ be a ring imbedded in a local ring $(Q, M)$ satisfying the following conditions.

(i) $Z(R)=M \cap R=Z(Q)$.

(ii) $\bar{R}=R / Z(R)$ is a right Ore domain.

Then $R$ has a local quotient ring $S$ which is a subring of $Q$.

Proof. Let $a$ be regular in $R$ and $b(\neq 0) \in M \cap R$. Then $\bar{a} \bar{n}=\bar{b} \bar{d}$ for any $n \in Z(R)$ and regular $d \in R$ since $\bar{n} \neq \overline{0} \neq \bar{b}$. Thus $a n-b d=m$ $\in M \cap R$. Since $Z(R)=M \cap R, a$ is a unit in $Q$ and so $a Q=Q$. Hence $m=a t, t \in R$ since otherwise $t \notin Z(Q), m$ is regular, a contradiction. This implies $a(n-t)=b d$. Thus $a$ and $b$ have a right multiple property. Now let $a$ be regular in $R$ and $b \in M \cap R$. By the condition (ii) we have $\bar{a} \bar{n}=\bar{b} \bar{d}, \bar{d}$ regular in $\bar{R}$. Here $d$ is regular in $R$ since $d \notin M \cap R$. Now $-a n+b d=e \in M \cap R$. Since $a \notin M, e=a t, t \in R$, otherwise $t$ and so $e$ becomes regular. So $a(n+t)=b d$. Thus we conclude that $R$ has a quotient ring $S$.

$S$ is a subring of $Q$ : Let $x \in S$. Then $x=r d^{-1}, d$ regular in $R$. Since $Z(R)=M \cap R, d \notin M \cap R$ i.e., $d$ is a unit in $Q$. Let $d^{-1 *}$ be the inverse of $d$ in $Q$. Then the mapping $r d^{-1} \rightarrow r d^{-1 *}$ identifies $S$ as a subring of $Q$.

$S$ is a local ring: If $x$ is a nonunit in $S$, then $x=r d^{-1}$, where $r$ is not regular in $R$. Hence $r \in Z(R)=M \cap R$ i.e., $x \in M$. Now if $x$ and $y$ are nonunits in $S$, then $x$ and $y \in M$ i.e., $x+y \in M$. Thus $x+y$ is a nonunit in $Q$. Since $S \subset Q, x+y$ is a nonunit in $S$. Thus we conclude that $S$ is a local ring.

3.3 Proposition. Let $S$ be a local quotient ring of $R$ and let $(T, M)$ be a local ring such that $R \subset T \subset S$ and $M \cap R=Z(R)$. Then $T=S$.

Proof. Let $s(\neq 0) \in S$. Then $s=a b^{-1}, a \in R$ and $b$ regular in $R$. Since $R \subset T$, and $M \cap R=Z(R), b \notin M$. So $b^{-1}$ exists and is in $T$. Hence $s \in T$.

The above result generalizes the idea of the quotient ring of an integral domain as the smallest subfield containing the integral domain.

4. Relation between $Z$ and $J$. The two important questions that arise on the assumption of $Z \subseteq J$ in 2.7 are:

(i) What are those rings $R$ with local quotient rings which satisfy the property $Z(R) \subseteq J(R)$ ? 
(ii) Is $Z \subseteq J$ a necessary condition for a ring to have a local quotient ring?

It can be seen in the following that uniform two-sided Noetherian rings provide the necessary examples to the class of rings specified in (i).

4.1 Definition. A ring is said to be right (left) uniform if every nonzero right (left) ideal has nonnull intersection with every nonzero right (left) ideal. A ring is said to be uniform if it is right and left uniform.

4.2 Theorem. Let $R$ be a uniform and two-sided Noetherian ring. Then $R$ has a two-sided quotient ring as a completely primary ring ( $a$ local ring with d.c.c. on right and left ideals).

Proof. Let $x^{r}$ and $x^{l}$ denote the right and left annihilators of $x$ respectively. By Corollary 6.2 of [4], the hypothesis implies that $\left\{x \mid x^{r} \neq 0\right\}$ and $\left\{x \mid x^{l} \neq 0\right\}$ are nil ideals. Hence $Z=N \subseteq J$. Thus $R$ satisfies regularity condition as defined in $[8$, p. 90] and hence $R$ has a right and left Artinian quotient ring [8, Theorem 5.5]. It then follows that this quotient ring is a local ring by 2.4 .

As regards the second question whether $Z \subseteq J$ is the necessary condition for a ring to have a local quotient ring, we present an example to show that this need not be the case.

4.3 Example. Let $R=D[[u, v]]$ be a formal power series in two variables $u$ and $v$ with coefficients in a field $D$ such that $u v=v u$. Since $D[[u, v]]=D[[u]][[v]], R$ is a local ring.

Elements of $R$ are of the form $\left(a_{00}+a_{01} u+\cdots\right)+\left(a_{10}+a_{11} u+\cdots\right) v$ $+\cdots+\left(a_{n 0}+a_{n 1} u+\cdots+\cdots\right) v^{n}+\cdots$. Suppose that $I$ is the ideal generated by $u^{i} v^{j}, i>1, j \geqq 1, i, j$ integers. Let $\bar{R}=R / I$. Elements of $\bar{R}$ are of the form $\left(a_{00}+a_{01} \bar{u}+\cdots\right)+\left(a_{10}+a_{11} \bar{u}\right) v+\cdots$ $+\left(a_{n 0}+a_{n 1} \bar{u}\right) \bar{v}^{n}+\cdots$. Consider $\bar{S}$, a polynomial ring with coefficients in $D$ in the nonzero terms $\bar{u}^{i} \bar{v}^{j}$.

Since the homomorphic image of a local ring is a local ring, $\bar{R}$ is a local ring and $\bar{S} \subset \bar{R}$ and $\bar{R}$ and $\bar{S}$ have the same identity 1 . Since $\bar{u}(\bar{u} \bar{v})=\overline{0}$, then $\bar{u} \in Z(\bar{S})$. Suppose $\bar{u} \in J(\bar{S})$. Then $1-\bar{u}$ has an inverse in $\bar{S}$. But the inverse of $1-\bar{u}$ is $1+\bar{u}+\bar{u}+\cdots$, which is not in $\bar{S}$. Hence $\bar{u} \notin J(\bar{S})$. Thus we conclude that $Z(\bar{S}) \Phi J(\bar{S})$.

Now we prove $Z(\bar{S})=Z(\bar{R}) \cap \bar{S}$. Evidently $Z(\bar{S}) \subseteq Z(\bar{R}) \cap \bar{S}$.

Assume $f \in Z(\bar{R})$ and

$$
\begin{aligned}
f= & \left(a_{00}+a_{01} \bar{u}+\cdots\right)+\left(a_{10}+a_{11} \bar{u}\right) \bar{v}+\cdots \\
& +\left(a_{n 0}+a_{n 1} \bar{u}\right) \bar{v}^{n}+\cdots
\end{aligned}
$$


Now $f g=0, g \neq 0$, where

$$
\begin{aligned}
g= & \left(b_{00}+b_{01} \bar{u}+\cdots\right)+\left(b_{10}+b_{11} \bar{u}\right) \bar{v}+\cdots \\
& +\left(b_{n 0}+b_{n 1} \bar{u}\right) \bar{v}^{n}+\cdots .
\end{aligned}
$$

Thus we can write

$g=B_{0}+B_{1} \bar{v}+B_{2} \bar{v}^{2}+\cdots$ where $B_{n}=\left(b_{n 0}+\cdots+b_{n 1} \bar{u}\right), n \neq 0$.

Let $a_{00} \neq 0$. Then by comparing the coefficients, we have

$$
\begin{aligned}
& a_{00} b_{00}=0 \\
& a_{00} b_{01}+a_{01} b_{00}=0 \\
& . \cdot . \cdot . \cdot . \cdot . \\
& a_{00} b_{0 n}+\cdots+a_{0 n} b_{00}=0
\end{aligned}
$$

Since the coefficients are in $D$, we have $b_{00}=\cdots=b_{0 n}=0$. Then by induction we have $b_{0 r}=0$ for all integral $r \geqq 0$. Thus, $B_{0}=0$.

By a similar argument, we can prove that a finite number of $B$ 's are zero. Then, by induction, we conclude that all $B$ 's are 0 . Thus $f g=0 \Rightarrow g=0$, a contradiction. Hence $a_{00}=0$.

Proceeding as above we can show that a finite number $a_{01}, \cdots, a_{0 n}$ will be zero and then, by induction it can be shown $a_{0 r}=0$ for all integral $r \geqq 0$. Thus $f=\left(a_{10}+a_{11} \bar{u}\right) \bar{v}+\cdots$. If $f \in Z(\bar{R}) \cap \bar{S}$, then $f=\left(a_{10}+a_{11} \bar{u}\right) \bar{v}+\cdots+\left(a_{n 0}+a_{n 1} \bar{u}\right) \bar{v}^{n}$. Hence, $f \bar{u}^{2}=0$. This implies $f \in Z(\bar{S})$. Thus $Z(\bar{R}) \cap \bar{S}=Z(\bar{S})$. Since $Z(\bar{S})$ is an ideal, by $2.2, S$ has a local quotient ring.

A simple modification obtained by considering a countable number of indeterminates in the above problem leads to an example of a nonNoetherian ring with a local quotient ring.

\section{REFERENCES}

1. S. A. Amitsur, Remarks on principal ideal rings, Osaka J. Math. 15 (1963),

2. P. M. Cohn, Non-commutative unique factorization domains, Trans. Amer. Math. Soc. 109 (1963), 313-331.

3. E. H. Feller and E. W. Swokowski, Reflective $N$-prime rings with the ascending chain condition, Trans. Amer. Math. Soc. 99 (1961), 264-284.

4. E. H. Feller, Noetherian modules and noetherian injective rings, Tôhoku Math. J. (2) 17 (1965), pp. 130-138.

5. A. W. Goldie, Lecture notes, Yale Univ., New Haven, Conn., 1961. 
6. - - The structure of prime rings, Proc. London Math. Soc. (3) 8 (1958), 589608.

7. - - - Semi-prime rings with maximum conditions, Proc. London Math. Soc. 10 (1960), 201-220.

8. I. N. Herstein, Topics in ring theory, Mathematical lecture notes, Univ. of Chicago, 1965.

9. Lance Small, Thesis, Univ. of Chicago, 1965.

10. O. F. G. Schilling, Ideal theory on open Riemann surfaces, Bull. Amer. Math. Soc. 52 (1946), 945-963.

11. T. D. Talintyre, Quotient rings with maximum condition on right ideals, J. London Math. Soc. 38 (1963), 439-450.

12. - - Quotient rings with minimum condition on right ideals, J. London Math. Soc. 41 (1966), 141-144.

S. V. University, Tirupati, India and Bowling Green State University 